\title{
Spur-Free Dynamic Range of Photonic Links Using Polarization Modulation
}

\author{
Thomas E. Darcie, Fellow, IEEE, Amol Moye, \\ Jeffrey D. Bull, Hiroshi Kato, Nicolas A. F. Jaeger
}

\begin{abstract}
The spur-free dynamic range (SFDR) of a novel microwave-photonic link, using a polarization mode-converter electro-optic modulator in a balanced output configuration, is characterized in this work. Common-mode intensity noise and optical-amplifier-induced beat noise are suppressed using a polarization-selective balanced optical receiver. In addition, thirdorder predistortion is used to reduce 2-tone intermodulation distortion by up to $20 \mathrm{~dB}$, further increasing the SFDR. Unlike the conventional approach using a dual-output Mach-Zehnder modulator, the complementary output signals are combined naturally as orthogonal polarizations into one transmission fiber.
\end{abstract}

Index Terms-Analog links, Intensity-noise suppression, Microwave-photonic links, Optical modulator, Polarization modulator, Polarization mode converter, Spur-free dynamic range

\section{INTRODUCTION}

$\mathrm{O}^{\mathrm{s}}$ PTICAL links for microwave communications, or microwave-photonic links (MWPL) have become an essential component in a variety of applications, including cable television networks, radar systems, and remote antenna systems. Therefore, extensive effort has gone into maximizing link performance [1,2]. Performance is bounded for weak signals by noise, which determines the minimum detectable signal, and for strong signals by nonlinear distortion, which limits the maximum transmitted signal. The spur-free dynamic range (SFDR) expresses the operating range between these two bounds.

Relative intensity noise (RIN) can be generated by the source laser, beating between the source laser and spontaneous emission generated by an optical amplifier, or multi-path interference from fiber connectors or doubleRayleigh scattering in the fiber. It has been shown [e.g., 2, 3, 4] that common-mode RIN (present at the input to the modulator) can be suppressed in optical links using balanced optical detection and a dual-output Mach-Zehnder (MZ) intensity modulator. By producing the difference between received complementary output signals in a balanced detector, common-mode RIN is cancelled, while the complementary modulated signals add. This has enabled the realization of MWPLs that have reduced RIN, but at the expense of two transmission fibers, a dual-output modulator, a balanced detector, and careful balancing of power levels.

Linearization of modulators is undertaken to mitigate the effects of nonlinear distortion on the SFDR of the link. Three primary types of modulator linearization have been explored over the last two decades - electronic predistortion [5], electro-optic feed-forward [6], and optical linearization of the modulator transfer function [7]. Predistortion can be implemented with simple nonlinear circuit elements and is the most widely used linearization technique. Electro-optic

This work was supported by National Sciences and Engineering Research Council, Canadian Institute for Photonic Innovation, and JGKB Photonics, Inc., Burnaby, BC, Canada.

T. E. Darcie is with University of Victoria, Victoria, BC, V8W 3P6A, Canada (phone: 250-721-8686; fax: 250-721-6052; e-mail: tdarcie@ uvic.ca).

A. Moye is with University of Victoria (amoye@uvic.ca).

J. D. Bull and H. Kato are with JGKB Photonics Inc, 221-4664 Lougheed Highway, Burnaby, BC, V5C 5T5, Canada (phone: 604-221-5452; fax: 604-221-5453; www.jgkb.com; jeff.bull@jgkb.com).

N. A. F. Jaeger is with the University of British Columbia, Vancouver, BC, V6T 1Z4, Canada (phone: 604-822-5673; fax: 604-822-5949; e-mail: nickj@ece.ubc.ca).

Photonic Applications in Nonlinear Optics, Nanophotonics, and Microwave Photonics,

edited by Roberto A. Morandotti, Harry E. Ruda, Jianping Yao, Proc. of SPIE Vol. 5971 , 59711V, (2005) - 0277-786X/05/\$15 - doi: 10.1117/12.628070 
feed-forward involves the detection of the difference between the desired and the actual transmitted intensity modulation, followed by the addition of a second optical signal that compensates this difference. While effective, this adds substantial complexity. Optically linearized modulators have been demonstrated successfully, but have yet to find widespread application.

In this paper we quantify the performance of an MWPL that uses a novel polarization-mode converter electrooptic polarization modulator (MCPM) [8] in a balanced configuration with a polarization-selective balanced receiver to cancel common-mode RIN, as first reported in [9]. Also, linearization of the modulator is performed through electronic predistortion to increase the SFDR. We describe the basic operation of the MCPM, link configuration, predistortion circuit, and characterize the performance of the link in terms of SFDR. We conclude that the performance of the MCPMbased link is comparable that of the MZ-based link. However, the MCPM may provide advantages in cost and physical size as it naturally combines the two complementary output signals as orthogonal polarizations in a single fiber.

\section{EXPERIMENT}

Figure 1 shows the basic MWPL configuration for RIN suppression and electronic predistortion, in both standard MZ and MCPM configurations. A single-waveguide mode-converter MCPM [8] was used to switch light between polarization states that lie on the great circle of the Poincare sphere that connects the horizontal $(\mathrm{H})$ and vertical (V) linear states and left (L) and right-hand (R) circular polarization states. Using a polarization controller and an input polarizer on the MCPM, linearly-polarized light was launched equally into each of two hybrid eigen-modes of the $\mathrm{AlGaAs} / \mathrm{GaAs}$ waveguide. These two modes are linearly polarized at approximately $45^{\circ}$ to the normal TE- and TM-like orientations. A voltage applied to the velocity-matched electrodes induces equal and opposite phase changes in these hybrid modes, resulting in ultrahigh-speed $(40 \mathrm{GHz})$ polarization modulation with low differential-group delay. A dual output lithium niobate MZ intensity modulator was used for comparison purposes. To suppress RIN, it was necessary to have equal powers in both output polarizations (or output ports of the MZ). To achieve this, and minimize second-order distortion, the modulators were biased at the point of $50 \%$ transmission (quadrature). Electronic predistortion was achieved by using a predistorter circuit that generates third-order distortion to a degree dependent on a bias voltage. This device is discussed in detail in Section 3.3.

A tunable laser was used as a laser source, coupled to an erbium-doped fiber amplifier (EDFA) (17 dBm output power), a tunable filter (TF) to eliminate signal-signal beat noise, and a polarization controller (PC). A maximum power of $14 \mathrm{dBm}$ was available at the input to the modulator at a wavelength of $1.55 \mu \mathrm{m}$. A single fiber connected the MCPM to a polarizing beam-splitter (PBS). The balanced detector (Discovery Semiconductors, Inc.) consisted of two high-speed InGaAs detectors $(18 \mathrm{GHz}$ bandwidth) with the common node matched to $50 \Omega$ and a specified common-mode suppression (no modulation) of $30 \mathrm{~dB}$. Noise and third-order 2-tone distortion were measured for a variety of optical received powers and RF drive powers at frequencies near 2.5 GHz. Standard 2-tone tests for linearity were performed using two synthesized RF sweep generators and an RF directional coupler.

\section{RESULTS AND DISCUSSION}

\subsection{Operation of the MCPM-based Link}

The MCPM provides an output that is very close to ideal polarization modulation (low residual intensity and phase modulation). Therefore, intensity modulation is generated entirely at the polarization beam splitter (PBS) preceding the detectors. Working in conjunction with the PC and PBS, the MCPM effectively creates a distributed MZ between the two orthogonal polarization modes. A bias point for this distributed MZ is set with the PC. The polarization state emerging from the PC can be adjusted to any point on a great circle that corresponds to equal powers exiting the two ports of the PBS; however, the trajectory traced out by the MCPM must also be oriented with the PC so that it passes through the two linear states of the PBS. Modulation of the MCPM voltage about such a bias point creates complementary intensity modulation in the two output arms of the PBS with maximum modulation depth. This intensity modulation exhibits the same sinusoidal transfer function as a conventional MZ, as is demonstrated in earlier work [9]. 


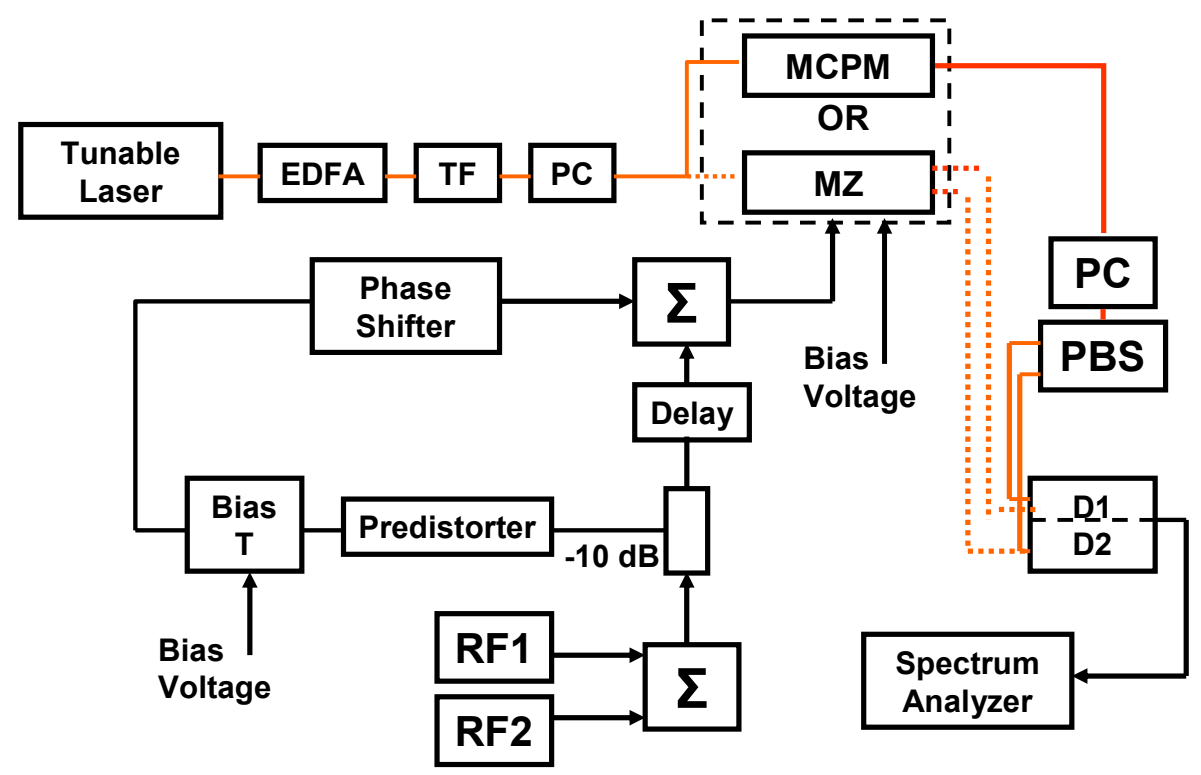

Figure 1. Experimental setup for intensity-noise suppression and electronic predistortion using mode-converter polarization modulator (MCPM) and Mach-Zehnder (MZ) modulators, showing tunable filter (TF), polarization controllers (PC), polarizing beamsplitter (PBS), and photodetectors (D1, D2).

\subsection{Suppression of RIN}

Considering the system shown in Figure 1, biased at the quadrature point, the output power of the MZ modulator, or MCPM following the PBS, can be written as:

$$
P_{Q o}=\frac{L_{e}}{2} P_{M Z i}(1+\sin \pi v),
$$

where $v$ is the modulation voltage normalized to the switching voltage $V_{\pi}, P_{M Z i}$ is the optical power at the input to the modulator, and $L_{e}$ is the excess loss. This results in a detected current in a single detector

$$
I_{Q}=\frac{R_{o} L L_{e}}{2} P_{M Z i}(1+\sin \pi v)=\frac{1}{2} I_{o}(1+\sin \pi v),
$$

where $L$ is the loss between the modulator and detector, $R_{o}$ is the detector responsivity $(\mathrm{A} / \mathrm{W})$, and $I_{o}$ is the photocurrent corresponding to $100 \%$ transmission of the modulator.

Signal Power: Assuming small-signal modulation of the form $v=v_{o} \cos \omega t$, the root-mean-square (rms) signal power (per ohm) is given by:

$$
P_{S_{Q}}=\frac{1}{2}\left(\frac{I_{o} \pi v_{O}}{2}\right)^{2} .
$$

Noise: Using an optical amplifier prior to the modulator results in signal-spontaneous beat noise that can be described by an additional component to the relative-intensity noise (RIN), described [9] by:

$$
R I N^{*}=R I N+2(1-1 / G) F h f / P_{a i},
$$

where $G$ is the amplifier power gain, $F$ the noise figure, $h f$ the photon energy, and $P_{a i}$ is the optical power at the input to the amplifier. The rms noise current from RIN* in a single detector, averaged over multiple RF cycles, is then due entirely to the average DC photocurrent $I_{o} / 2$; 


$$
\sigma_{R I N^{*}}=I_{o} \sqrt{B \cdot R I N^{*}} / 2 .
$$

In determining the total mean-square noise power from RIN*, one can consider the effects of intensity-noise suppression in the balanced detector. This results in [10]:

$$
\sigma_{R I N^{*}}^{2}=I_{o}^{2}\left(B \cdot R I N^{*}\right)\left(S_{o}^{2}+2-2 e^{-2 \mu^{2}}\right) / 4=I_{o}^{2}\left(B \cdot R I N^{*}\right) S^{2} / 4
$$

$S_{O}$ is an amplitude suppression factor that accounts for the common-mode suppression in the absence of modulation.

Common-mode rejection in terns of RF noise power $\left({S_{o}}^{2}\right)$ may exceed $30 \mathrm{~dB}$. For increasing modulation, the degree of suppression $S$ becomes limited by the instantaneous difference in power received by the two detectors, as described by the additional terms in Equation (6) involving the normalized root-mean square modulation index $\mu$, where $\mu^{2}=m^{2} N / 2$, and $m$ is the modulation index for each of $\mathrm{N}$ channels. Alternatively, $\mu^{2}$ is the variance of the modulated output-intensity probability-density function. For $\mu$ greater than $20 \%$, suppression of noise power $\left(S^{2}\right)$ cannot be greater than approximately $10 \mathrm{~dB}$.

Uncorrelated shot noise is generated in each detector such that the total mean-square shot noise power is:

$$
\sigma_{\text {shot }}^{2}=2 \cdot 2 e \frac{I_{o}}{2} B \text {. }
$$

Finally, noise generated by the receiver can be described by an equivalent mean-square receiver noise current. For high received powers, shot noise and RIN typically dominate.

Figure 2 illustrates the noise suppression achieved using our balanced MCPM-based link. Nearly identical results are obtained for the MZ using the two output fibers. In order to implement optimal RIN suppression by this method, the phase and optical power in the two arms should be perfectly balanced. In our setup, we were able to balance phase and power corresponding to a suppression factor $(S)$ of 0.1 . This corresponds to suppression being limited by the modulation depth of $2.7 \%$, as measured on a sampling oscilloscope, and using Equation (6). As seen from the graph, RIN is suppressed nearly to the limit imposed by shot noise. For larger modulation indices, we would expect to see less suppression and a departure from purely shot-noise-limited performance.

\subsection{Third-Order Intermodulation Cancellation}

The predistortion linearization technique used in this work is based on creating a distorted signal at the input to the modulator to cancel the modulator nonlinearity. Our predistorter circuit is configured to have equal and opposite nonlinearity to that resulting from the sinusoidal transfer function of the modulator. Figure 3 shows the schematic of the third-order predistortion circuit. The bias voltage applied to the output port of the circuit varies the pre-bias applied equally to the two opposing low-barrier Schottky diodes, controlling the amount of third-order distortion generated. Figure 4 illustrates the response of the predistorter to various RF powers at different bias voltages. For reference, the RF power of the fundamental signals through the predistorter is approximately $-25 \mathrm{dBm}$ at an input power of $-14 \mathrm{dBm}$. It can be seen that the predistorter creates substantial third-order distortion, relative to the nonlinearity of the modulators, over a wide range of input RF power, and the magnitude of distortion can be adjusted over tens of $\mathrm{dB}$ using the bias voltage. This provides a convenient way to adjust the magnitude of the predistortion signal to cancel the modulator nonlinearity. 


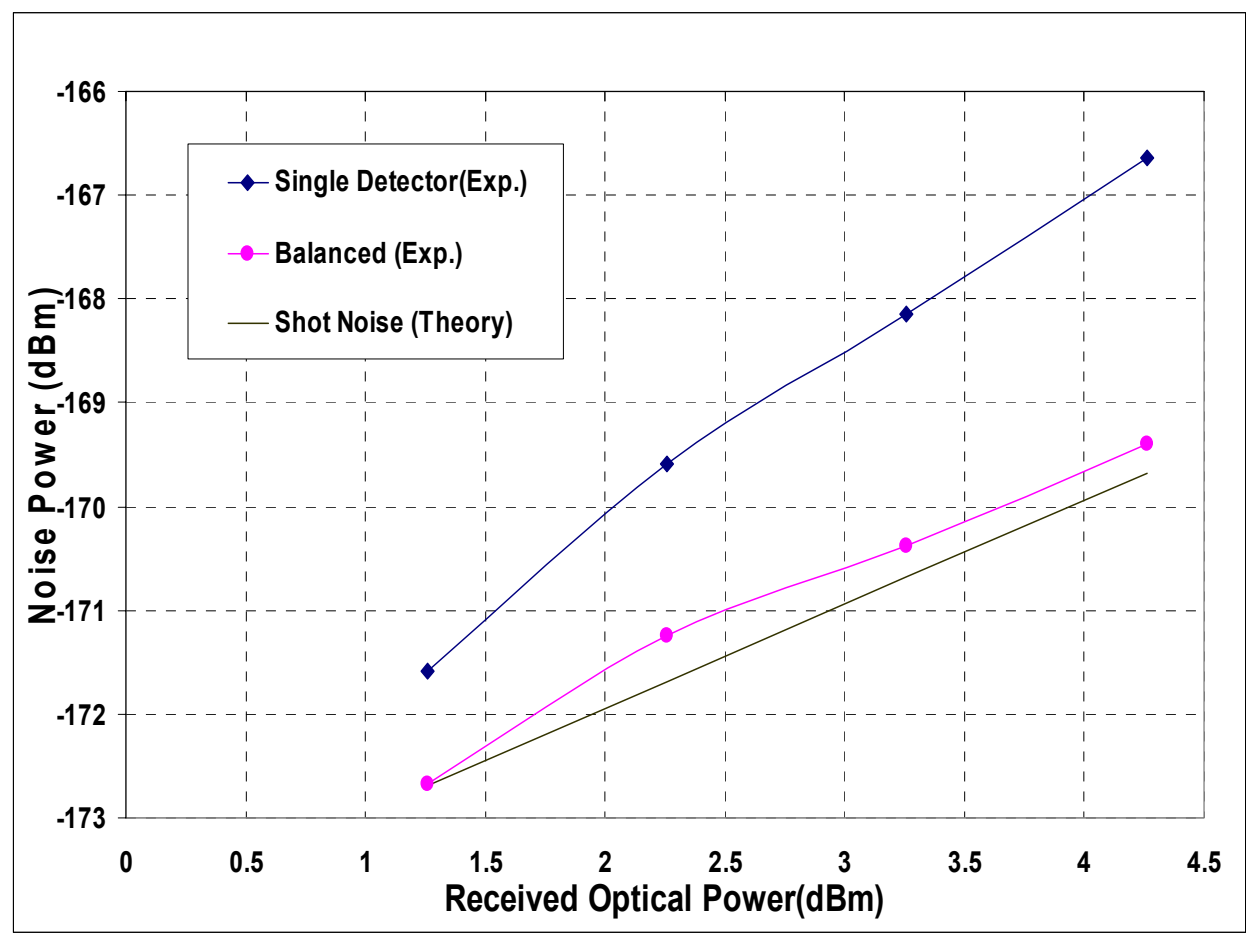

Figure 2. Demonstration of RIN suppression in the MCPM-based link by balanced detection.

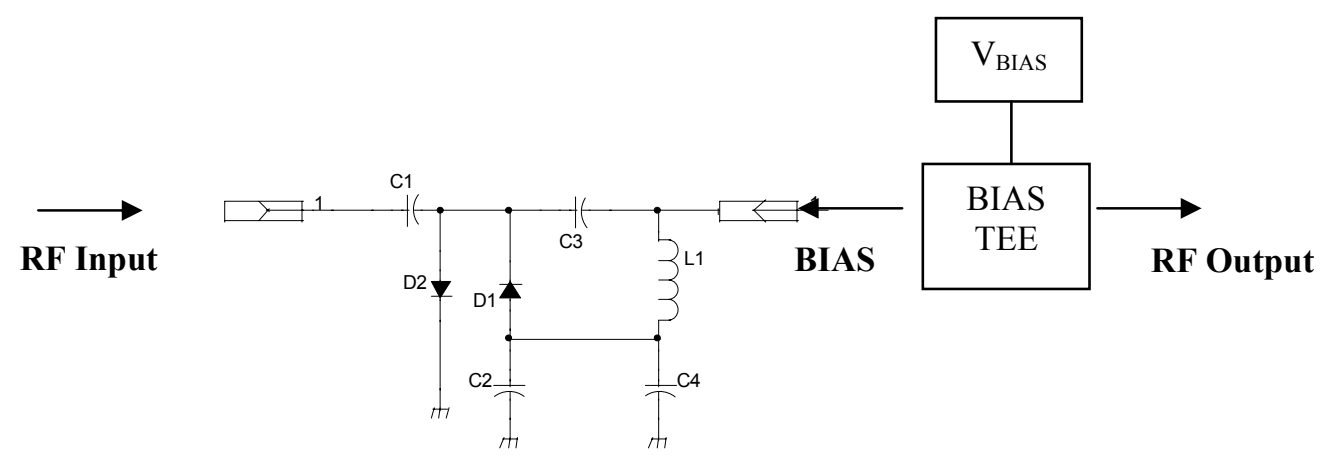

Figure 3. Schematic of the predistortion circuit. 


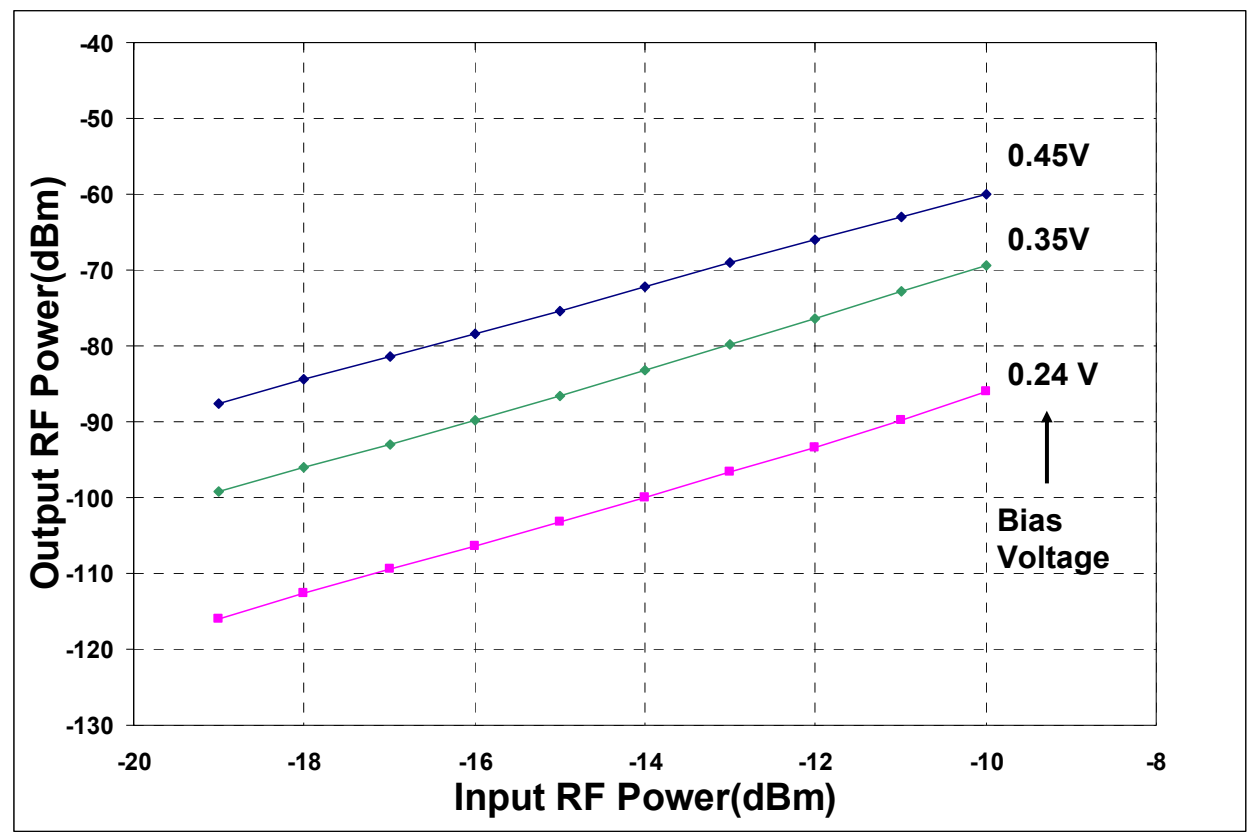

Figure 4. Response of the output 2-tone intermodulation product from the predistorter to various RF inputs at different bias voltages.

As shown in the experimental setup in Figure 1, two RF tones are combined using an in-phase power combiner and a portion of the signal is fed to the predistorter input via a $10 \mathrm{~dB}$ power tap. Third-order cancellation is achieved by adjusting the predistorter bias voltage such that the third-order distortion generated electronically by the predistortion circuit is equal but opposite to the third-order distortion generated in the output optical intensity of the modulator in response to the input RF signal. The output of the predistorter is combined with the RF input to the MCPM after undergoing a phase shift of 180 degrees. This is achieved by controlling the phase of the predistorted signal using an RF phase shifter. Maximum cancellation is obtained for perfectly matched amplitudes and phase of the third-order intermodulation products. As can be seen from Figure 5, third-order cancellation of up to $20 \mathrm{~dB}$ can be achieved for lower modulation indices $(5 \% \sim 8 \%)$. However, cancellation becomes less pronounced for increased modulation depths, and for input powers greater than $6 \mathrm{dBm}$, the predistorter becomes ineffective. This dependence on modulation index relates to the requirement that the third-order distortion generated by both the modulator and predistorter remain in close balance over the range of operating power. However, strong third-order distortion occurring at high modulation index also results in compression of the fundamental, and this compression may be slightly different for the two nonlinearities. We could adjust the amplitude of the predistortion signal to cancel the MCPM third-order distortion at any particular high input power, but this cancellation would exist over a narrow range of input power only. At lower modulation index, maximum cancellation results in an increase in the SFDR by $6.67 \mathrm{~dB}$, corresponding to a reduction in third-order distortion of $20 \mathrm{~dB}$.

Using the combination of intensity-noise suppression and third-order predistortion, we are able to achieve a (third-order) SFDR of $104.7 \mathrm{~dB} / \mathrm{Hz}^{2 / 3}$ at a total receiver optical power (for both detectors) of $4.3 \mathrm{dBm}$. While not remarkable in absolute terms, this is respectable given the total received power. Limitations to the SFDR are receiver noise, optical power, and third-order distortion. Optical power levels are insufficient to generate sufficient shot noise to dominate the noise from our balanced receiver (matched to $50 \Omega$ with a shunt resistance). Transformer-coupling the detectors to the $50 \Omega$ transmission line would help reduce the contribution from receiver noise. Additional optical power would also increase RF signal power. Our balanced detector has a maximum rated detected power of $10 \mathrm{dBm}$ per 
detector. Therefore, the use of an EDFA with approximately $8 \mathrm{~dB}$ higher gain could improve the SFDR by approximately $8 \mathrm{~dB}$. Finally, refinement to the predistorter circuit could in principle reduce the third-order distortion further. However, achieving the exact amplitude and phase balance required to achieve better linearity over a range of frequencies and RF power levels is challenging, and a $3 \mathrm{~dB}$ improvement in third-order distortion results in only a $1 \mathrm{~dB}$ improvement in SFDR. Hence we would not expect substantial improvements to come from improved predistortion.

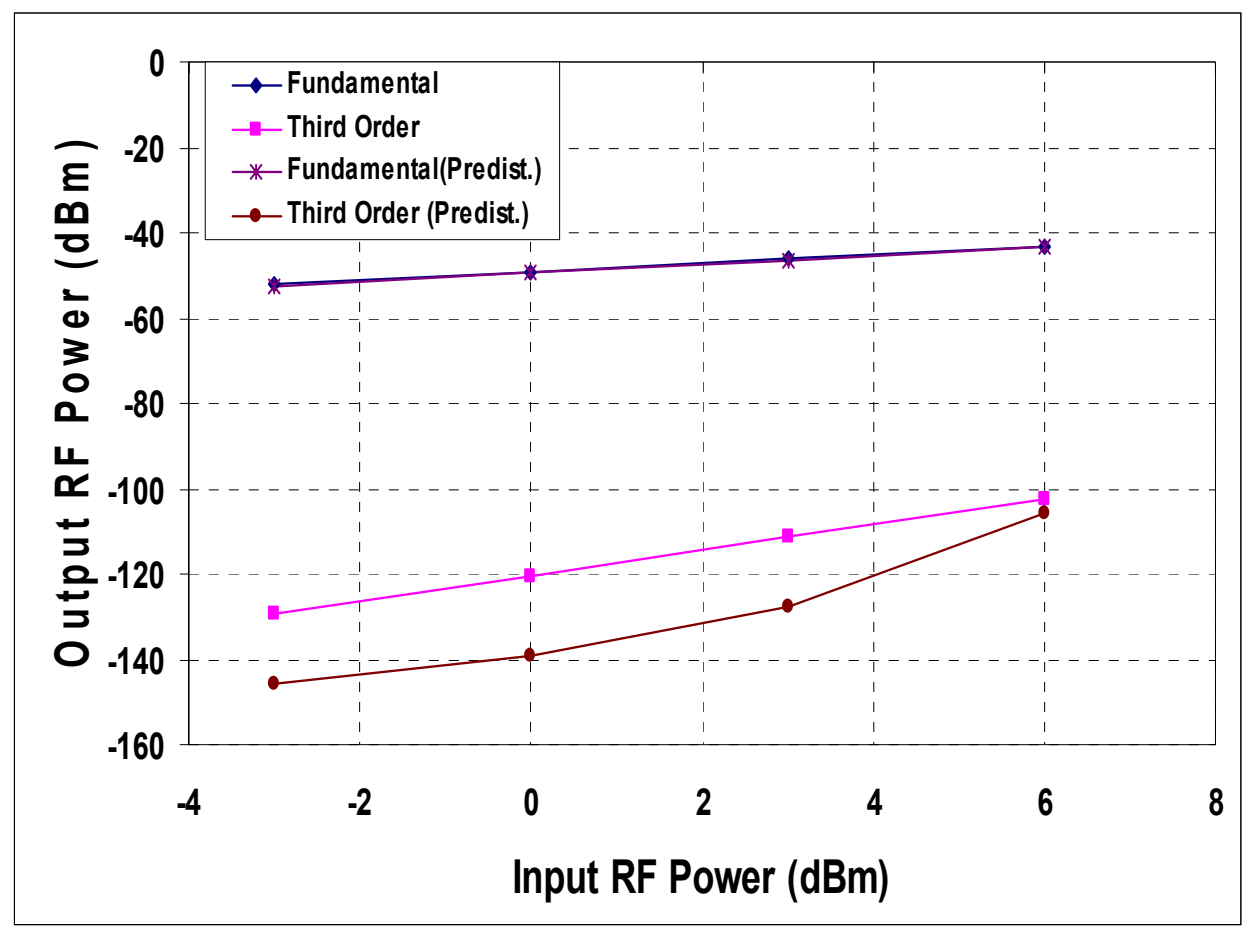

Figure 5. Demonstration of cancellation of third-order distortion by electronic predistortion.

\section{Summary}

We have demonstrated an increase in SFDR in a novel balanced microwave-photonic link using a polarization modulator. Both the signal and its complement are transmitted as orthogonal polarizations, then separated and differenced in a balanced detector. Balanced detection by this method offers the advantages of using a device with only one output fiber and one transmission fiber, unlike the standard dual-output Mach Zehnder, while obtaining high levels of common-mode noise suppression. The disadvantage is that a polarization splitter must be used prior to balanced detection. This tradeoff may favor the proposed approach in applications where weight, physical size, or fiber management are constrained. In addition, electronic third-order predistortion is used to increase the SFDR by compensating for the sinusoidal transfer functions of the modulator. We achieve a $20 \mathrm{~dB}$ reduction in third-order distortion using a simple and broadband circuit, and the reduction can be achieved without adjustment of predistorter amplitude or phase over a wide range of input RF power. These techniques, as well as increasing the available optical transmit power to the limit tolerated by the photodetectors, can extend the spur-free dynamic range in future microwave photonic links.

\section{REFERENCES}

[1] C. H. Cox III, G. E. Betts, and L. M. Johnson, "An analytic and experimental comparison of direct and external modulation in analog fiber-optic links," EEE Trans. Microwave Theory and Techniques, vol.38, pp. 501-509, May 1990.

[2] L. T. Nichols, K. J. Williams, and R. D. Esman, "Optimizing the ultrawide-band photonic link," IEEE Trans. Microwave Theory and Techniques, vol. 45, pp. 1384-1389, August 1997. 
[3] E. I. Ackerman, S. Wanuga, J. MacDonald, and J. Prince, "Balanced receiver external modulation fiber-optic link architecture with reduced noise figure," Microwave Symposium Digest, IEEE MTT-S, Atlanta, GA, vol. 2, pp. 723-726, 14-18, June 1993.

[4] S. Mathai, F. Cappelluti, T. Jung, D. Novak, R. B. Waterhouse, D. Sivco, A. Y. Cho, G. Ghione, M. C. Wu, "Experimental demonstration of a balanced electroabsorption modulated microwave photonic link," IEEE Trans. Microwave Theory and Techniques, Vol. 49, pp. 1956-1961, Oct. 2001.

[5] T. E. Darcie, G. E. Bodeep, "Lightwave subcarrier CATV transmission systems", IEEE Transactions on Microwave Theory and Techniques, Volume 38, Issue 5, pp. 524 - 533, May 1990.

[6] T. Iwai, K. Sato, K. Suto, K.," Signal distortion and noise in AM-SCM transmission systems employing the feedforward linearized MQW-EA external modulator", Journal of Lightwave Technology, Volume 13, Issue 8, pp. 1606 - 1612, Aug. 1995

[7] G. E. Betts, "Linearized modulator for suboctave-bandpass optical analog links," IEEE Transactions on Microwave Theory and Techniques, Volume 42, Issue 12, Part 1-2, pp. 2642 - 2649, Dec. 1994.

[8] F. Rahmatian, N. A. F. Jaeger, R. James, E. Berolo, "An ultrahigh-speed AlGaAs-GaAs polarization converter using slow-wave coplanar electrodes," IEEE Photonics Technology Letters, Vol.10, pp. 675 - 677, May 1998.

[9] T. E. Darcie, R. Paiam, A. Moye, J. D. Bull, H. Kato, N. A. F. Jaeger, "Intensity-noise suppression in microwave-photonic links using polarization modulation," IEEE Photonic Technology Letters, Vol. 17, No. 9, Sept. 2005.

[10] T. E. Darcie, A. Moye, "Modulation-dependent limits to intensity-noise suppression in microwave photonic links," accepted for publication, IEEE Photonic Technology Letters, 2005. 